\title{
The Study of Misclassification Probability in Discriminant Model of Pattern Identification for Stroke
}

\author{
Mi Mi Ko ${ }^{1}$ and Honggie Kim ${ }^{2}$ \\ ${ }^{1}$ KM Fundamental Research Division, Korea Institute of Oriental Medicine, Daejeon 305-811, Republic of Korea \\ ${ }^{2}$ Department of Information and Statistics, Chungnam National University, Daejeon 305-764, Republic of Korea
}

Correspondence should be addressed to Honggie Kim; honggiekim@cnu.ac.kr

Received 23 October 2015; Revised 12 January 2016; Accepted 15 February 2016

Academic Editor: Chris Zaslawski

Copyright (c) 2016 M. M. Ko and H. Kim. This is an open access article distributed under the Creative Commons Attribution License, which permits unrestricted use, distribution, and reproduction in any medium, provided the original work is properly cited.

\begin{abstract}
Background. Pattern identification (PI) is the basic system for diagnosis of patients in traditional Korean medicine (TKM). The purpose of this study was to identify misclassification objects in discriminant model of PI for improving the classification accuracy of PI for stroke. Methods. The study included 3306 patients with stroke who were admitted to 15 TKM hospitals from June 2006 to December 2012. We derive the four kinds of measure $(D, R, S$, and $C$ score) based on the pattern of the profile graphs according to classification types. The proposed measures are applied to the data to evaluate how well those detect misclassification objects. Results. In 10-20\% of the filtered data, misclassification rate of $C$ score was highest compared to those rates of other scores (42.60\%, $41.15 \%$, resp.). In $30 \%$ of the filtered data, misclassification rate of $R$ score was highest compared to those rates of other scores $(40.32 \%)$. And, in $40-90 \%$ of the filtered data, misclassification rate of $D$ score was highest compared to those rates of other scores. Additionally, we can derive the same result of $C$ score from multiple regression model with two independent variables. Conclusions. The results of this study should assist the development of diagnostic standards in TKM.
\end{abstract}

\section{Introduction}

Due to the development of modern medicine, the average lifespan for human beings is anticipated to rise beyond 85 years of age within the following 20 years [1]. In the meantime, since the rate of aging in South Korea is expected to surge up to $35.1 \%$ by 2050 , ranking 2 nd in the world close to Japan (37.7\%), geriatric diseases and the health of the elderly have emerged as one of the most critical social problems of improving the quality of life in the future [2]. In particular, stroke is one of the representative geriatric diseases, along with dementia. Personal and social insecurities caused by the disease have continued to grow. In addition, stroke ranks as the top mortality risk to Koreans among the single diseases and contributes to more than $70 \%$ of the in-patients at traditional Korean medical hospitals [3, 4]. In traditional Korean medicine (TKM), specific or nonspecific symptoms of patients are diagnosed by observing, listening, asking, and feeling their pulse under the diagnostic system of pattern identification (PI) in order to determine the cause, nature, treatment method, and treatment drugs of a disease [5-7]. This PI diagnosis collects specific or nonspecific symptoms of patients and classifies them into one of the hundreds of symptom classes. It is the essential core technology forming the backbone of diagnosis and treatment in oriental medicine. However, the PI diagnosis holds limited objectivity and reproducibility due to the lack of standardized measurement indices, and objectification problems have always arisen with respect to personal deviations among TKM physicians based on their knowledge and experience [6-8].

As the necessity for the standardization of diagnostic systems has recently come to the fore, studies have been underway to objectify diagnosis.

In the study titled "Fundamental Study for the Standardization and Objectification of Pattern Identification in Traditional Korean Medicine for Stroke (SOPI-Stroke)," which was conducted over 9 years from 2005 to 2013, the Korea Institute of Oriental Medicine (KIOM) proposed a standardization plan for PI/syndrome differentiation of stroke, established stroke PI diagnostic indices, built a database system relating 
TABLE 1: Results using the classification of discriminant model.

\begin{tabular}{|c|c|c|c|c|c|c|}
\hline & & \multicolumn{5}{|c|}{ Classification result $N(\%)$} \\
\hline & & QD & DP & YD & $\mathrm{FH}$ & Total \\
\hline \multirow{5}{*}{ Physician's diagnosis } & QD & $498(66.94)$ & $115(15.46)$ & $95(12.77)$ & $36(4.84)$ & $744(22.50)$ \\
\hline & DP & $118(10.61)$ & $783(70.41)$ & $69(6.21)$ & $142(12.77)$ & $1112(33.64)$ \\
\hline & YD & $70(14.64)$ & $55(11.51)$ & $276(57.74)$ & $77(16.11)$ & $478(14.46)$ \\
\hline & $\mathrm{FH}$ & $46(4.73)$ & $147(15.12)$ & $127(13.07)$ & $652(67.08)$ & $972(29.40)$ \\
\hline & Total & $732(22.14)$ & $1100(33.27)$ & $567(17.15)$ & $907(27.44)$ & $3306(100.00)$ \\
\hline
\end{tabular}

QD: Qi deficiency pattern; DP: Dampness-phlegm pattern; YD: Yin deficiency pattern; FH: Fire-heat pattern.

to TKM clinical technologies by setting up a clinical index database, and founded a scientific basis for stroke and PI by discovering stroke and PI biological indices, to which the latest research methods, such as OMICS, were applied. Studies were carried out to discover biological indices that could be helpful to stroke prevention by finding out what the stroke risk factors were [9-16].

Consequently, the purpose of this study was to identify misclassification objects in discriminant model of PI for improving the classification accuracy of PI for stroke patients. Although current TKM PI diagnostic tools for stroke were developed after several years of research and prepared for public release, the tools still need corrections and modifications in many aspects [17-19]. In this study, the key topics for discussion involve appropriate statistical methods to reduce the probability of diagnostic misclassification.

\section{Methods}

2.1. Subjects. The study included 3306 patients with stroke who were admitted to 15 oriental medical university hospitals from June 2006 to December 2012. Each patient provided informed consent to undergo procedures that were approved by the respective institutions' Institutional Review Boards (IRB). Informed consent of all the study patients was obtained after a thorough explanation of the details. We enrolled stroke patients for enrollment within 30 days of the onset of their symptoms, provided that their diagnosis was confirmed by an imaging diagnosis such as computerized tomography (CT) or magnetic resonance imaging (MRI). Patients with traumatic stroke such as subarachnoid, subdural, and epidural hemorrhage were excluded from the study.

2.2. Measured Variables. Each patient was seen by two experts at the same department within each site. All experts who were well trained in standard operation procedures (SOPs) were participating in this study. The experts had at least three years of clinical experiences with stroke after finishing regular college education about TKM for six years. The examination parameters were extracted from parts of a case report form (CRF) for the standardization of stroke diagnosis that had been developed by an expert committee organized by the KIOM $[7,11,12]$.

2.2.1. The Korean Standard PI for Stroke-3. PI process for differentiating stroke with four TKM types: the Fire-heat
(FH) pattern, Dampness-phlegm (DP) pattern, Yin deficiency (YD) pattern, and Qi deficiency (QD) pattern [11, 12]. The FH pattern is characterized by any symptom of heat or fire that is contracted externally or engendered internally. The DP pattern is characterized by impeding Qi movement and its turbidity, heaviness, stickiness, and downward-flowing properties. The QD pattern is characterized by qi deficiency with diminished internal organ function, which is marked by shortness of breath, lassitude, listlessness, spontaneous sweating, a pale tongue, and a weak pulse. The YD pattern is characterized by yin deficiency with diminished moistening and the inability to restrain yang, which is usually manifested as fever [7, 9-13, 20]. The Korean Standard PI for Stroke- 3 consists of 44 clinical indices and each clinical index belongs to its respective PI (Supplemental Table 1, in Supplementary Material available online at http://dx.doi.org/10.1155/2016/1912897).

2.3. Statistical Methods. After determining 12 different types of misclassification through discriminant analysis, we plotted it on the profile graphs according to types. And then we derive the four kinds of measure $(D, R, S$, and $C$ score) based on the pattern analysis of the profile graphs. The proposed measures are applied to the stroke data to evaluate how well those detect misclassification objects.

2.3.1. Types of Misclassification. According to the results from the discriminant model classification, 2,209 patients posted correct classifications out of the total of 3,306 patients (66.82\%) (Table 1). Out of the 3,306 patients, 1,097 were misclassified (33.2\%) and the misclassification types are summarized in Table 2. To analyze the misclassification types, 44 clinical indices of the Korean Standard PI for Stroke-3 were grouped into four upper-class variables (QD, DP, YD, and $\mathrm{FH}$ pattern indices). In addition, the average and standard deviation of each upper-class variable was used to attain standardized scores, after which the misclassification types were analyzed (Figure 1).

2.3.2. The Profile Graphs. With 12 misclassification types and 4 correct classification types categorized by the discriminant analysis, the profile graphs were drawn. Specifically, two of the 4 patterns were selected and the correct classification types and misclassification types for each pattern were collected from the TKM physicians and divided. For instance, as described in Figure 2, patients applicable to two misclassification types (FHQD and QDFH) were grouped together. 
TABLE 2: The mean values of the standardized scores for upper-class variables according to misclassification type.

\begin{tabular}{lcccccc}
\hline & Types of misclassification & $N(\%)$ & $Z_{\mathrm{QD}}$ & $Z_{\mathrm{DP}}$ & $Z_{\mathrm{YD}}$ \\
\hline 1 & DPFH $^{\#}$ & $142(12.94)$ & -0.565 & -0.113 & -0.251 & 0.648 \\
2 & DPQD & $118(10.76)$ & 1.004 & -0.001 & -0.312 & -0.492 \\
3 & DPYD & $69(6.29)$ & 0.118 & -0.060 & 0.902 \\
4 & FHDP & $147(13.40)$ & -0.426 & 0.610 & -0.114 & 0.085 \\
5 & FHQD & $46(4.19)$ & 0.907 & -0.494 & -0.233 & 0.096 \\
6 & FHYD & $127(11.58)$ & -0.291 & -0.596 & 0.956 & 0.184 \\
7 & QDDP & $115(10.48)$ & 0.111 & 0.605 & -0.394 & -0.456 \\
8 & QDFH & $36(3.28)$ & 0.075 & -0.500 & -0.373 \\
9 & QDYD & $95(8.66)$ & 0.512 & -0.487 & 0.808 \\
10 & YDDP & $55(5.01)$ & -0.229 & 0.529 & -0.153 \\
11 & YDFH & $77(7.02)$ & -0.393 & -0.525 & -0.299 \\
12 & YDQD & $70(6.38)$ & 0.914 & -0.492 & 0.133 \\
\hline & & $1097(100.00)$ & 0.067 & -0.063 & 0.240 \\
\hline
\end{tabular}

QD: Qi deficiency pattern; DP: Dampness-phlegm pattern; YD: Yin deficiency pattern; FH: Fire-heat pattern; DPFH ${ }^{\#}$ : physician's diagnosis- Dampness-phlegm pattern, classification result, Fire-heat pattern; $Z_{\mathrm{QD}}$ : the standardized scores for upper-class variables according to Qi deficiency pattern; $Z_{\mathrm{DP}}$ : the standardized scores for upper-class variables according to Dampness-phlegm pattern; $Z_{\mathrm{YD}}$ : the standardized scores for upper-class variables according to Yin deficiency pattern; $Z_{\mathrm{FH}}$ : the standardized scores for upper-class variables according to Fire-heat pattern.

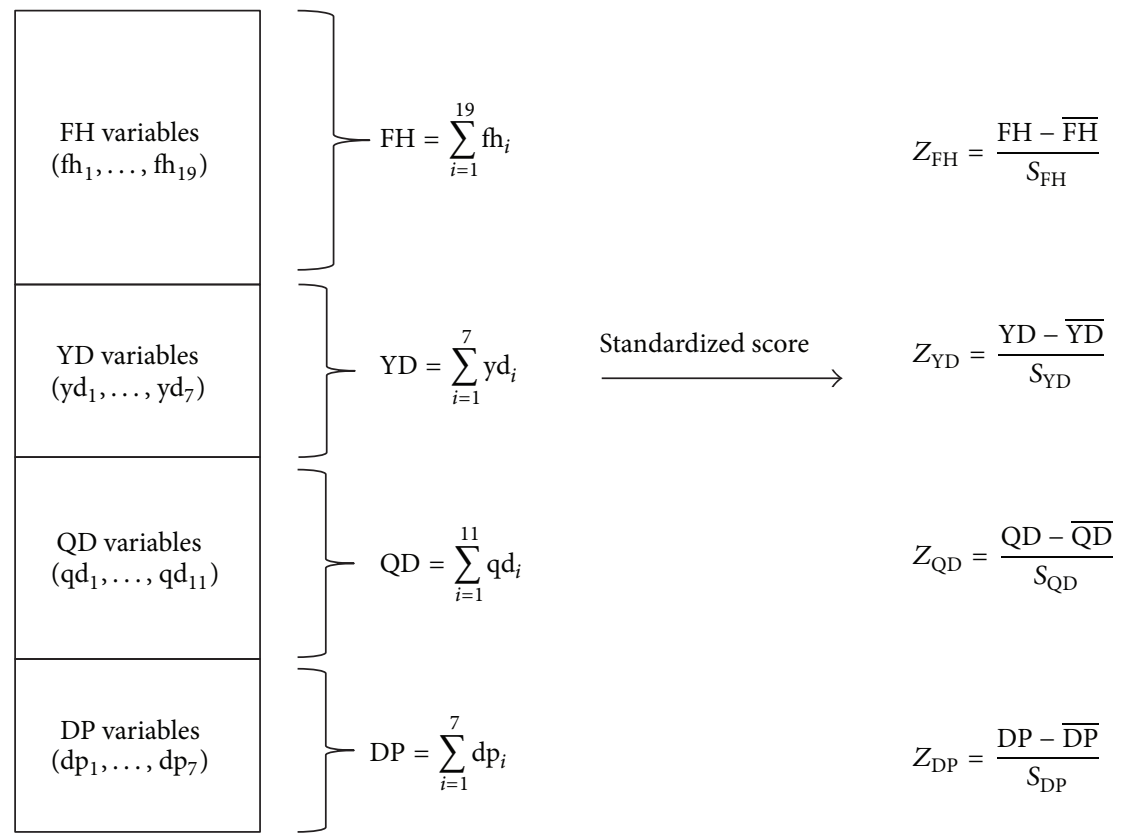

FIGURE 1: Process of grouping of explanatory variables and standardized scores generation. The mean and standard deviation of each upperclass variable were used to attain standardized scores, after which the misclassification types were analyzed. QD: Qi deficiency pattern; DP: Dampness-phlegm pattern; YD: Yin deficiency pattern; FH: Fire-heat pattern.

Next, the upper-class variable scores of each patient were used to draw a profile plot. At this point, it was critical to arrange the pattern scores of correct classification on the edges and those of the other two pattern scores inside. The profile graphs of the misclassification types (FHQD, $\mathrm{QDFH}$, etc.) and the correct classification types (e.g., FH, QD, YD, and DP) are depicted in Figures 2-7 and the relevant statistics are in Table 3. As illustrated in Figures 2-7, two misclassification types demonstrate a U-shaped pattern and correct classification types an L-shaped or flipped-L-shaped pattern.

2.3.3. Derived Four Measures (D, R, S, and C Scores). In the profile graphs, misclassification observations in most of the 6 cases displayed a bathtub or U-shaped pattern since pattern scores corresponding to actual patterns would be relatively high and the misclassification of a pattern is highly probable if relatively higher scores were observed in the other pattern. 


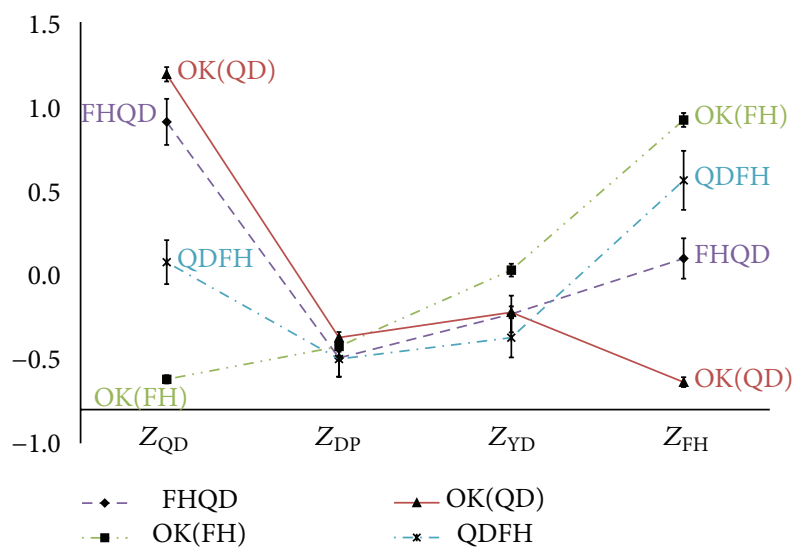

FIGURE 2: The profiles graphs of the $\mathrm{FH}$ and $\mathrm{QD} . Z_{\mathrm{FH}}$ : the standardized scores for upper-class variables according to Fireheat pattern; $Z_{\mathrm{QD}}$ : the standardized scores for upper-class variables according to Qi deficiency pattern; $Z_{\mathrm{DP}}$ : the standardized scores for upper-class variables according to Dampness-phlegm pattern; $Z_{\mathrm{YD}}$ : the standardized scores for upper-class variables according to Yin deficiency pattern; OK: the correct classification types.

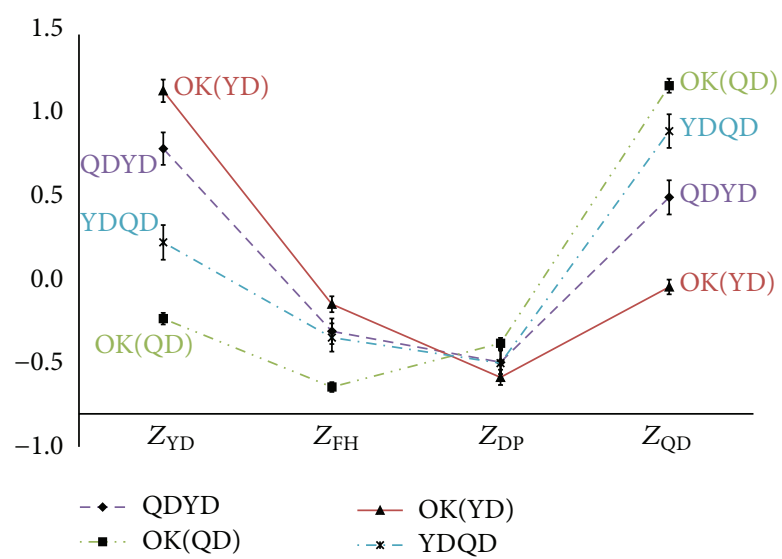

FIGURE 3: The profiles graphs of the $\mathrm{QD}$ and YD. $Z_{\mathrm{FH}}$ : the standardized scores for upper-class variables according to Fireheat pattern; $Z_{\mathrm{QD}}$ : the standardized scores for upper-class variables according to Qi deficiency pattern; $Z_{\mathrm{DP}}$ : the standardized scores for upper-class variables according to Dampness-phlegm pattern; $Z_{\mathrm{YD}}$ : the standardized scores for upper-class variables according to Yin deficiency pattern; OK: the correct classification types.

In the meantime, correct classification observations showed an L-shaped (or flipped-L-shaped) pattern. Although actual patterns are unknown due to the lack of direct diagnoses from TKM physicians, if a new patient establishes a bathtubshaped profile simply with 4 upper-class pattern scores (obligatory two high scores and two low scores), this patient is likely to be misclassified through the future discriminant model. Criteria were designed to assess how close a pattern score profile would be to a bathtub shape through various arrangements and simple calculations of the four pattern scores and applied to already discriminated data. By doing so, comparison was conducted to investigate how much misclassification was estimated and how much discrimination rates

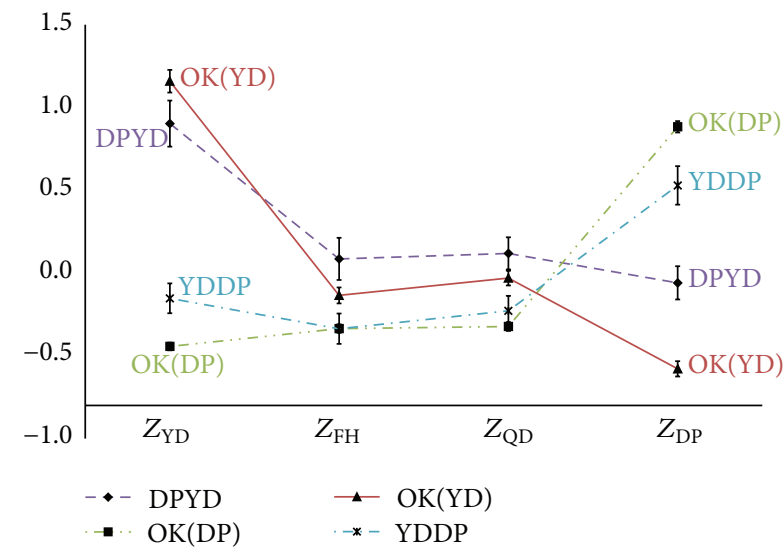

FIGURE 4: The profiles graphs of the DP and YD. $Z_{\mathrm{FH}}$ : the standardized scores for upper-class variables according to Fireheat pattern; $Z_{\mathrm{QD}}$ : the standardized scores for upper-class variables according to Qi deficiency pattern; $Z_{\mathrm{DP}}$ : the standardized scores for upper-class variables according to Dampness-phlegm pattern; $Z_{\mathrm{YD}}$ : the standardized scores for upper-class variables according to Yin deficiency pattern; OK: the correct classification types.

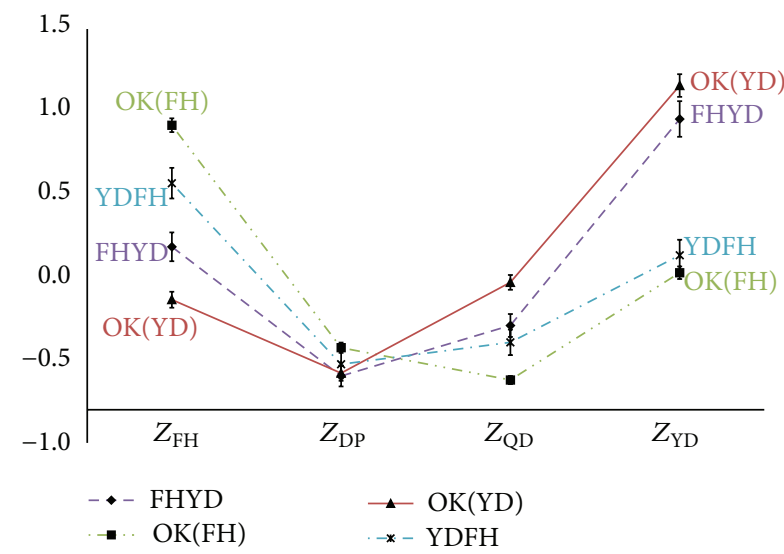

Figure 5: The profiles graphs of the $\mathrm{FH}$ and YD. $Z_{\mathrm{FH}}$ : the standardized scores for upper-class variables according to Fireheat pattern; $Z_{\mathrm{QD}}$ : the standardized scores for upper-class variables according to Qi deficiency pattern; $Z_{\mathrm{DP}}$ : the standardized scores for upper-class variables according to Dampness-phlegm pattern; $Z_{\mathrm{YD}}$ : the standardized scores for upper-class variables according to Yin deficiency pattern; OK: the correct classification types.

improved when the estimated misclassification observations were eliminated beforehand.

(1) D Score. Analyzing correct classification and misclassification types with profile graphs, the $D$ value was derived considering that a difference between the maximum value $Z_{(1)}$ and the second-largest value $Z_{(2)}$ of misclassification was smaller than that of correct classification, and classification by the value was attempted (Figure 8). Namely, under the hypothesis that the smaller the $D$ value was, the closer the profile graph was to a bathtub shape and the higher the probability of the respective observations corresponding to misclassification was, the $D$ values were applied to the clinical stroke data. 


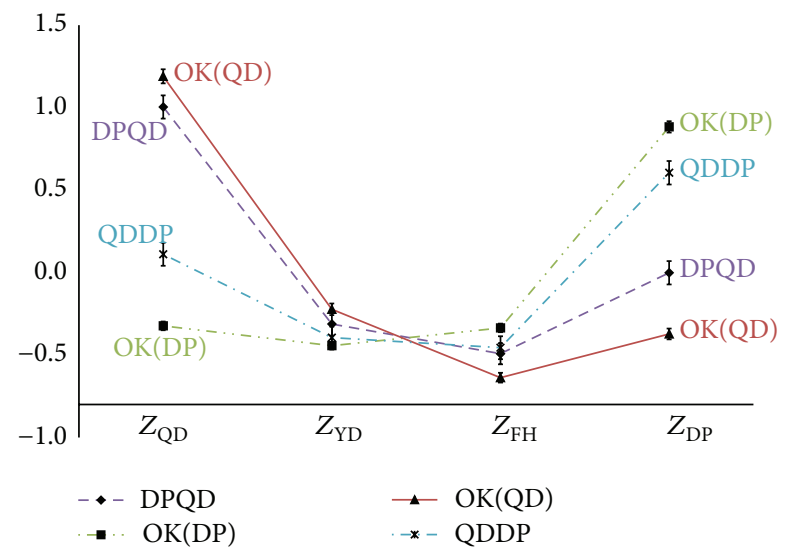

FIgURE 6: The profiles graphs of the DP and QD. $Z_{\mathrm{FH}}$ : the standardized scores for upper-class variables according to Fireheat pattern; $Z_{\mathrm{QD}}$ : the standardized scores for upper-class variables according to Qi deficiency pattern; $Z_{\mathrm{DP}}$ : the standardized scores for upper-class variables according to Dampness-phlegm pattern; $Z_{\mathrm{YD}}$ : the standardized scores for upper-class variables according to Yin deficiency pattern; OK: the correct classification types.

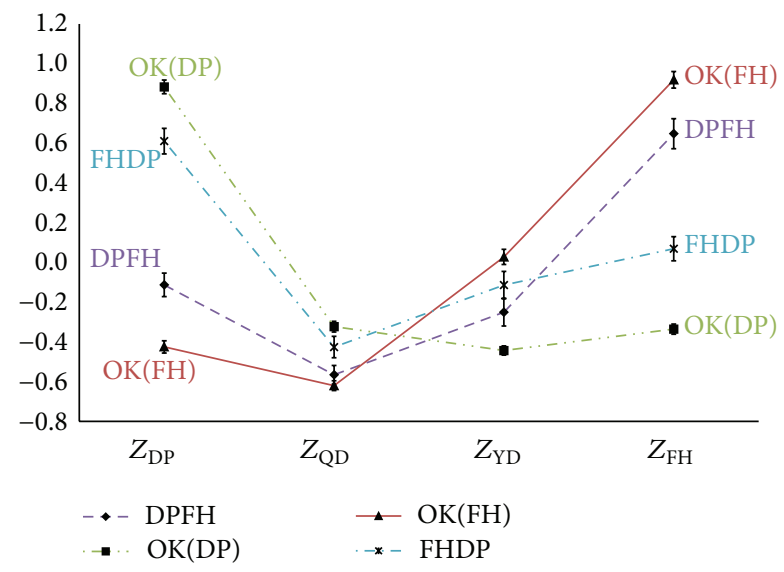

FIgURE 7: The profiles graphs of the DP and FH. $Z_{\mathrm{FH}}$ : the standardized scores for upper-class variables according to Fireheat pattern; $Z_{\mathrm{QD}}$ : the standardized scores for upper-class variables according to Qi deficiency pattern; $Z_{\mathrm{DP}}$ : the standardized scores for upper-class variables according to Dampness-phlegm pattern; $Z_{\mathrm{YD}}$ : the standardized scores for upper-class variables according to Yin deficiency pattern; OK: the correct classification types.

After sorting the data by the $D$ value in descending order and investigating the frequency and rates of misclassification over $10 \%$ intervals (Figure 9), the misclassification probability of the $10 \%(N=331)$ filtered data reached $40.79 \%\left(N_{m}=\right.$ 135, Mean $\left._{m}=0.058\right)$, which was $7.61 \% p$ higher than the previously calculated misclassification probability $(33.18 \%)$ of the total data. The misclassification probabilities of the data filtered from $20 \%$ to $90 \%$ were lower than that of the $10 \%$ filtered data but higher than that of the total data $(33.18 \%)$. In the data filtered at $10 \%, 20 \%, 40 \%$, and $50 \%$, average $D$ values of the misclassifications and correct classifications were barely different from each other, even though the average $D$ values of the misclassifications tended to be higher than those of the correct classifications. In the other data groups, the average $D$ values of the correct classifications were higher than those of the misclassifications (Table 4). Meanwhile, examining the frequencies and rates of the correct classifications in the data selected for $D$ values, the misclassification probability of the correct classifications in the $90 \%(N=2975)$ selected data recorded $67.66 \%$ $\left(N_{c}=2013, \%\right.$ of $\left.N_{m}=32.34 \%\right)$, which was $0.86 \% p$ higher than those of the previously calculated correct classifications $(66.8 \%)$ of the total data. In the $80 \%(N=2645)$ selected data, the misclassification probabilities of correct classifications reached $68.28 \%\left(N_{c}=1806, \%\right.$ of $\left.N_{m}=31.72 \%\right)$, which was $0.62 \% p$ higher than those in the $90 \%$ selected data. In the data selected from $70 \%$ to $10 \%$, the correct classifications gradually increased (Table 4 ).

(2) $R$ Score. Analyzing correct classification and misclassification types with profile graphs, the $R$ value was derived considering that a difference between the maximum value $Z_{(1)}$ and the minimum value $Z_{(4)}$ of misclassification was smaller than that of correct classification, and classification by the value was attempted (Figure 10). Namely, under the hypothesis that the larger the $R$ value was, the closer the profile graph was to an L-shaped or flipped-L-shaped pattern, and the higher the probability of the respective observations corresponding to correct classification was the $R$ values were applied to the clinical stroke data in the same way as previously (Table 5).

(3) S Score. Analyzing correct classification and misclassification types with profile graphs, the $S$ value was derived considering that the second-largest value $Z_{(2)}$ of misclassification was higher than that of correct classification, and classification by the value was attempted (Figure 11). Namely, under the hypothesis that the larger the $S$ value was, the closer the profile graph was to a bathtub (or $\mathrm{U}$ ) shape and the higher the probability of the respective observations corresponding to misclassification was, the $S$ values were applied to the clinical stroke data. In this case, the frequency and rates of misclassification over $10 \%$ intervals were investigated after sorting the data by the $S$ value in ascending order (Table 6).

(4) C Score. Analyzing correct classification and misclassification types with profile graphs, the $C$ value was derived considering that a difference between the sum of $Z_{(1)}$ and $Z_{(2)}$ and the sum of $Z_{(3)}$ and $Z_{(4)}$ of misclassification was larger than that of correct classification, and classification by the value was attempted (Figure 12). Namely, under the hypothesis that the larger the $C$ value was, the closer the profile graph was to a bathtub (or $U$ ) shape, the higher the probability of the respective observations corresponding to misclassification was, the $C$ values were applied to the clinical stroke data in the same way as previously (Table 7).

\section{Results}

3.1. Estimated Misclassification Probability and Discrimination Rate according to Proposed Four Scores. Table 8 summarizes the misclassification probabilities after the data was sorted 
TABLE 3: Summary of $Z$ scores according to the profile graphs for PI classification types.

\begin{tabular}{|c|c|c|c|c|c|c|}
\hline & \multirow{2}{*}{ Classification types } & \multirow{2}{*}{$N$} & \multicolumn{4}{|c|}{$Z$ scores $($ mean $\pm \mathrm{SE})$} \\
\hline & & & $Z_{\mathrm{QD}}$ & $Z_{\mathrm{DP}}$ & $Z_{\mathrm{YD}}$ & $Z_{\mathrm{FH}}$ \\
\hline \multirow{5}{*}{ FH, QD classification types } & FHQD & 46 & $0.907 \pm 0.137$ & $-0.494 \pm 0.110$ & $-0.233 \pm 0.109$ & $0.097 \pm 0.120$ \\
\hline & $\mathrm{OK}(\mathrm{FH})$ & 652 & $-0.620 \pm 0.025$ & $-0.425 \pm 0.031$ & $0.028 \pm 0.038$ & $0.919 \pm 0.042$ \\
\hline & $\mathrm{OK}(\mathrm{QD})$ & 498 & $1.189 \pm 0.043$ & $-0.372 \pm 0.033$ & $-0.223 \pm 0.035$ & $-0.637 \pm 0.030$ \\
\hline & QDFH & 36 & $0.075 \pm 0.130$ & $-0.500 \pm 0.107$ & $-0.373 \pm 0.118$ & $0.560 \pm 0.175$ \\
\hline & Total & 1232 & $0.189 \pm 0.034$ & $-0.408 \pm 0.022$ & $-0.095 \pm 0.025$ & $0.249 \pm 0.034$ \\
\hline \multirow{5}{*}{ QD, YD classification types } & QDYD & 95 & $0.513 \pm 0.103$ & $-0.487 \pm 0.072$ & $0.808 \pm 0.099$ & $-0.300 \pm 0.078$ \\
\hline & $\mathrm{OK}(\mathrm{QD})$ & 498 & $1.189 \pm 0.043$ & $-0.372 \pm 0.033$ & $-0.223 \pm 0.035$ & $-0.637 \pm 0.030$ \\
\hline & $\mathrm{OK}(\mathrm{YD})$ & 276 & $-0.031 \pm 0.045$ & $-0.579 \pm 0.046$ & $1.159 \pm 0.068$ & $-0.135 \pm 0.048$ \\
\hline & YDQD & 70 & $0.914 \pm 0.102$ & $-0.493 \pm 0.090$ & $0.240 \pm 0.105$ & $-0.337 \pm 0.085$ \\
\hline & Total & 939 & $0.742 \pm 0.034$ & $-0.454 \pm 0.024$ & $0.322 \pm 0.036$ & $-0.433 \pm 0.025$ \\
\hline \multirow{5}{*}{ DP, YD classification types } & DPYD & 69 & $0.118 \pm 0.097$ & $-0.060 \pm 0.101$ & $0.903 \pm 0.139$ & $0.085 \pm 0.127$ \\
\hline & $\mathrm{OK}(\mathrm{DP})$ & 783 & $-0.323 \pm 0.027$ & $0.883 \pm 0.034$ & $-0.443 \pm 0.024$ & $-0.336 \pm 0.026$ \\
\hline & $\mathrm{OK}(\mathrm{YD})$ & 276 & $-0.031 \pm 0.045$ & $-0.579 \pm 0.046$ & $1.159 \pm 0.068$ & $-0.135 \pm 0.048$ \\
\hline & YDDP & 55 & $-0.229 \pm 0.090$ & $0.529 \pm 0.116$ & $-0.153 \pm 0.090$ & $-0.336 \pm 0.092$ \\
\hline & Total & 1183 & $-0.225 \pm 0.022$ & $0.471 \pm 0.032$ & $0.022 \pm 0.032$ & $-0.264 \pm 0.023$ \\
\hline \multirow{5}{*}{ FH, YD classification types } & FHYD & 127 & $-0.291 \pm 0.069$ & $-0.597 \pm 0.063$ & $0.956 \pm 0.108$ & $0.184 \pm 0.087$ \\
\hline & $\mathrm{OK}(\mathrm{FH})$ & 652 & $-0.620 \pm 0.025$ & $-0.425 \pm 0.031$ & $0.028 \pm 0.038$ & $0.919 \pm 0.042$ \\
\hline & $\mathrm{OK}(\mathrm{YD})$ & 276 & $-0.031 \pm 0.045$ & $-0.579 \pm 0.046$ & $1.159 \pm 0.068$ & $-0.135 \pm 0.048$ \\
\hline & YDFH & 77 & $-0.393 \pm 0.077$ & $-0.525 \pm 0.086$ & $0.133 \pm 0.095$ & $0.568 \pm 0.093$ \\
\hline & Total & 1132 & $-0.424 \pm 0.022$ & $-0.489 \pm 0.023$ & $0.415 \pm 0.034$ & $0.555 \pm 0.032$ \\
\hline \multirow{5}{*}{ DP, QD classification types } & DPQD & 118 & $1.004 \pm 0.071$ & $-0.001 \pm 0.071$ & $-0.312 \pm 0.070$ & $-0.492 \pm 0.064$ \\
\hline & $\mathrm{OK}(\mathrm{DP})$ & 783 & $-0.323 \pm 0.027$ & $0.883 \pm 0.034$ & $-0.443 \pm 0.024$ & $-0.336 \pm 0.026$ \\
\hline & $\mathrm{OK}(\mathrm{QD})$ & 498 & $1.189 \pm 0.043$ & $-0.372 \pm 0.033$ & $-0.223 \pm 0.035$ & $-0.637 \pm 0.030$ \\
\hline & QDDP & 115 & $0.111 \pm 0.070$ & $0.605 \pm 0.071$ & $-0.395 \pm 0.067$ & $-0.456 \pm 0.069$ \\
\hline & Total & 1514 & $0.311 \pm 0.028$ & $0.380 \pm 0.027$ & $-0.357 \pm 0.019$ & $-0.456 \pm 0.018$ \\
\hline \multirow{5}{*}{ DP, FH classification types } & DPFH & 142 & $-0.565 \pm 0.047$ & $-0.113 \pm 0.059$ & $-0.251 \pm 0.069$ & $0.648 \pm 0.076$ \\
\hline & $\mathrm{OK}(\mathrm{DP})$ & 783 & $-0.323 \pm 0.027$ & $0.883 \pm 0.034$ & $-0.443 \pm 0.024$ & $-0.336 \pm 0.026$ \\
\hline & $\mathrm{OK}(\mathrm{FH})$ & 652 & $-0.620 \pm 0.025$ & $-0.425 \pm 0.031$ & $0.028 \pm 0.038$ & $0.919 \pm 0.042$ \\
\hline & FHDP & 147 & $-0.426 \pm 0.054$ & $0.610 \pm 0.064$ & $-0.114 \pm 0.068$ & $0.069 \pm 0.061$ \\
\hline & Total & 1724 & $-0.464 \pm 0.017$ & $0.283 \pm 0.026$ & $-0.221 \pm 0.020$ & $0.254 \pm 0.026$ \\
\hline
\end{tabular}

PI: pattern identification; QD: Qi deficiency pattern; DP: Dampness-phlegm pattern; YD: Yin deficiency pattern; FH: Fire-heat pattern; OK: the correct classification types; $Z_{\mathrm{QD}}$ : the standardized scores for upper-class variables according to Qi deficiency pattern; $Z_{\mathrm{DP}}$ : the standardized scores for upper-class variables according to Dampness-phlegm pattern; $Z_{\mathrm{YD}}$ : the standardized scores for upper-class variables according to Yin deficiency pattern; $Z_{\mathrm{FH}}$ : the standardized scores for upper-class variables according to Fire-heat pattern.
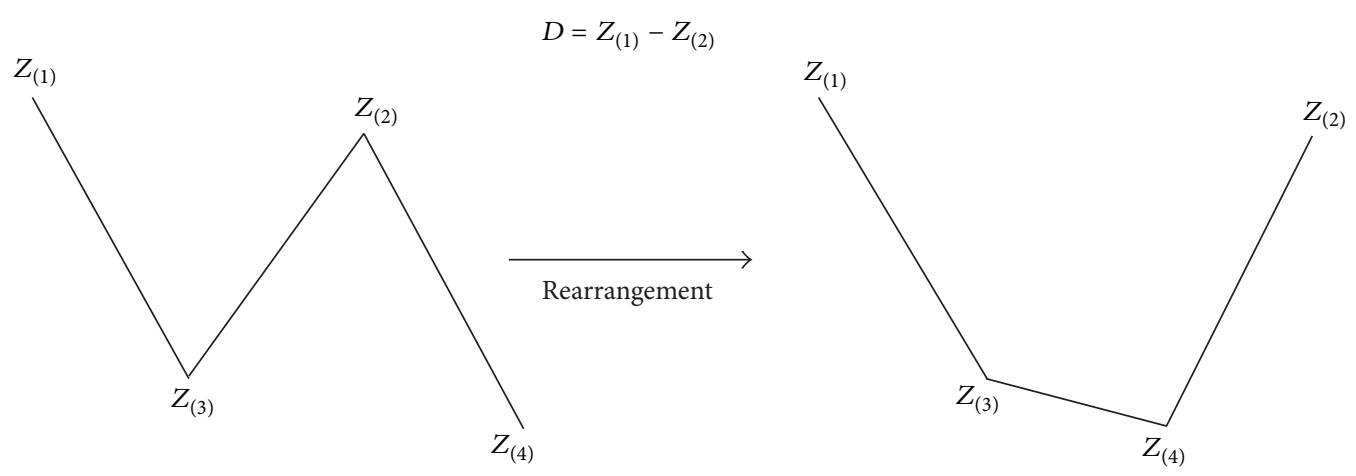

Figure 8: Derived $D$ values based on the pattern analysis of the profile graphs. Under the hypothesis that the smaller the $D$ value was, the closer the profile graph was to a bathtub (or U) shape, and the higher the probability of the respective observations corresponding to misclassification was. 


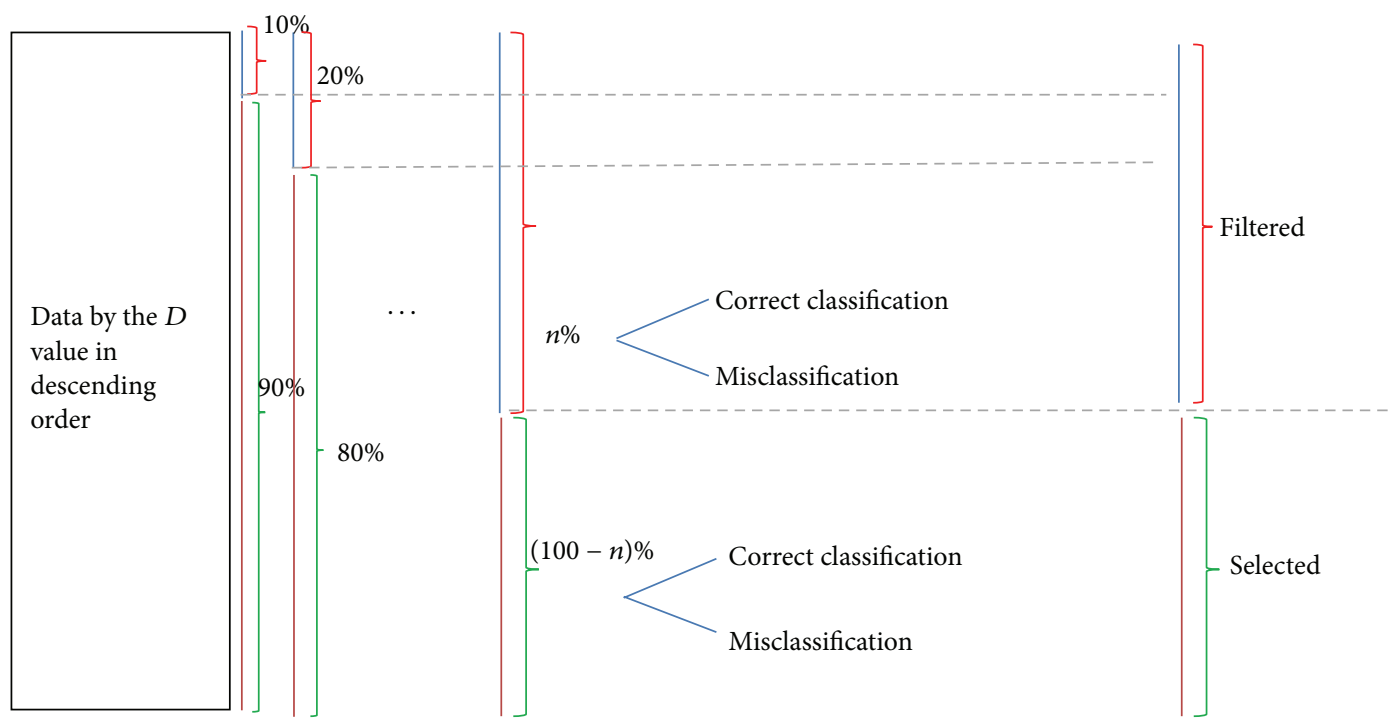

Figure 9: Data filtering and selection method. Data were ranged according to each four measures ( $D, R, S$, and $C$ values) in descending or ascending order by increasing data by $10 \%$ intervals.

according to the 4 criteria and investigating the misclassification probability over $10 \%$ intervals. If the data were filtered $10-20 \%$, the $C$ score marked $42.60 \%$ and $41.15 \%$, respectively, indicating the highest misclassification probability among the criteria. If the data were filtered $30 \%$, the $R$ score stands at $40.32 \%$ and the $C$ score at $39.92 \%$. If the data were filtered $40 \sim 90 \%$, the misclassification probability of the $D$ score was the highest.

For the data previously selected by 4 scores $(D, R, S$, and $C$ ), discrimination rates were compared. Having the 4 $\mathrm{QD}, \mathrm{DP}, \mathrm{YD}$, and $\mathrm{FH}$ patterns set as reaction variables for the entire clinical stroke data and 44 clinical indices of the Korean Standard PI for Stroke-3 as independent variables, the discriminant analysis was conducted to calculate the discrimination accuracy (Table 9). If the data were selected at $90 \%$, the discrimination rate of the $D$ score increased to $68.2 \%$, which was the largest increase among the four scores. If the data were selected at $80 \%$, the $C$ score reached $69.0 \%$, making the largest increase. If the data were selected at $70 \%$, the $R$ score posted $70.0 \%$, demonstrating the largest increase in the discrimination rate among the four scores. If the data were selected at $60-10 \%$, the $D$ score recorded the largest increase in the discrimination rate among the four scores.

\subsection{Similarities between Secondary Curvature \\ Function and C Score}

3.2.1. Curvature Created by $Z_{(1)}, Z_{(2)}, Z_{(3)}$, and $Z_{(4)}$ Scores. First of all, assume four scores, $Z_{(1)}, Z_{(2)}, Z_{(3)}$, and $Z_{(4)}$, as dependent variables observed in the $x$ values (e.g., $1,2,3$, and 4) having equal intervals, as shown in the profile graphs. In addition, assume that $Z_{(1)}$ is a dependent variable when $x=$ $1, Z_{(2)}$ when $x=4, Z_{(3)}$ when $x=2$, and $Z_{(4)}$ when $x=3$. This assumption is illustrated in Figure 13.
3.2.2. Estimation of Secondary Curvature. Considering the quadratic curve regression model passing through the four points $\left(1, Z_{(1)}\right),\left(2, Z_{(3)}\right),\left(3, Z_{(4)}\right)$, and $\left(4, Z_{(2)}\right), Y=\beta_{0}+$ $\beta_{1} X+\beta_{2} X^{2}+\epsilon$, the coefficient of $\beta_{2}$ is the secondary curvature value that we wanted. Namely, the larger the $\beta_{2}$ is, the stronger the bathtub shape becomes, boosting the misclassification probability. Assuming that the estimates of $\beta_{0}, \beta_{1}$, and $\beta_{2}$ are $b_{0}, b_{1}$, and $b_{2}$, these estimates satisfy the following normal equation [21]:

$$
\left(X^{\prime} X\right) b=X^{\prime} Y
$$

Here

$$
\begin{aligned}
& X=\left[\begin{array}{lll}
1 & 1 & 1^{2} \\
1 & 2 & 2^{2} \\
1 & 3 & 3^{2} \\
1 & 4 & 4^{2}
\end{array}\right], \\
& b=\left[\begin{array}{l}
b_{0} \\
b_{1} \\
b_{2}
\end{array}\right], \\
& Y=\left[\begin{array}{l}
Z_{(1)} \\
Z_{(2)} \\
Z_{(3)} \\
Z_{(4)}
\end{array}\right] .
\end{aligned}
$$

According to Neter et al. [21], a general two-variable regression model,

$$
Y_{i}=\beta_{0}+\beta_{1} X_{i 1}+\beta_{2} X_{i 2}+\epsilon_{i}
$$




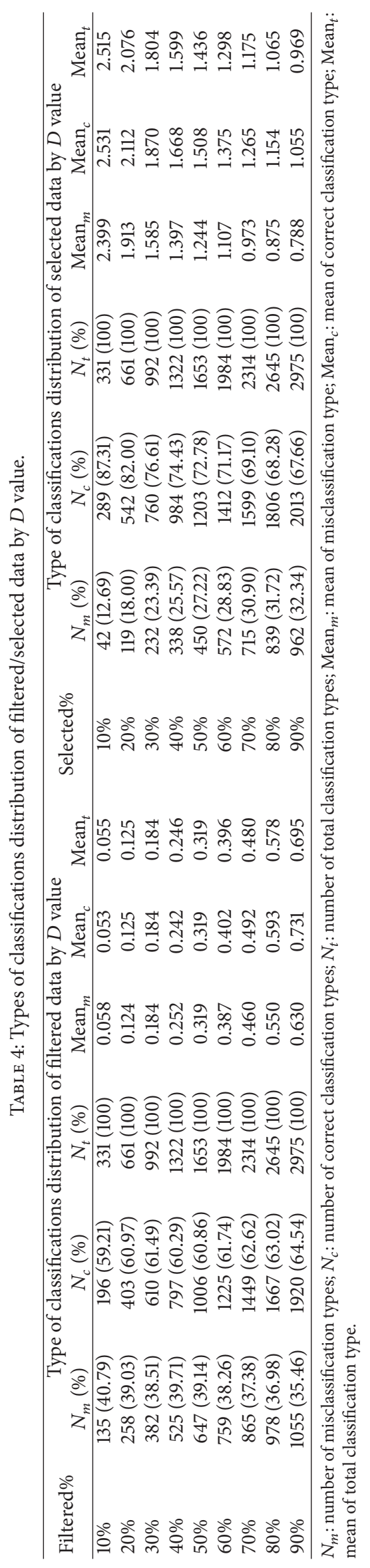




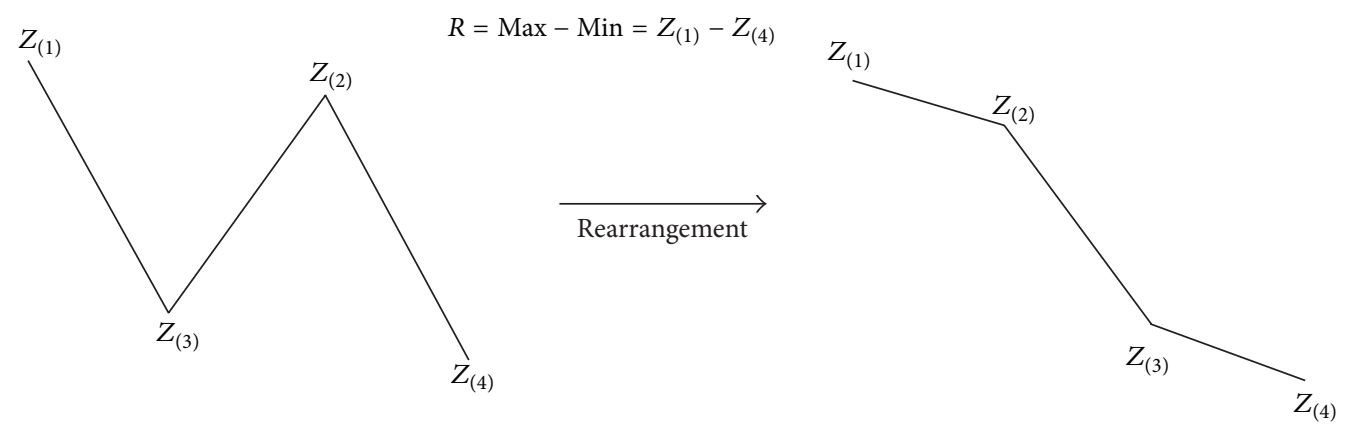

FIGURE 10: Derived $R$ values based on the pattern analysis of the profile graphs. Under the hypothesis that the larger the $R$ value was, the closer the profile graph was to an L-shaped or flipped-L-shaped pattern, the higher the probability of the respective observations corresponding to correct classification was.

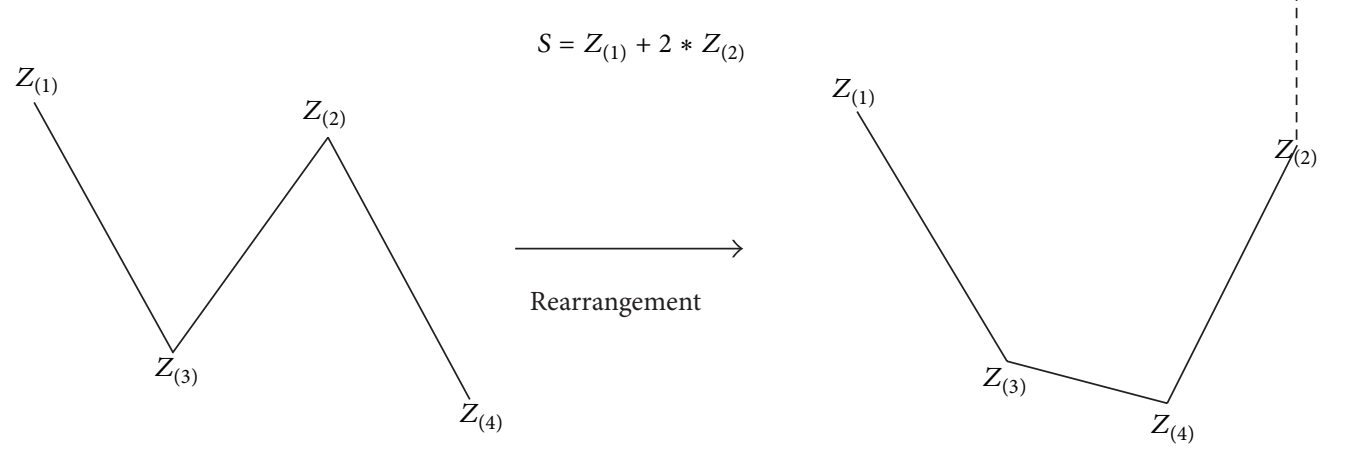

FIGURE 11: Derived $S$ values based on the pattern analysis of the profile graphs. Under the hypothesis that the larger the $S$ value was, the closer the profile graph was to a bathtub (or U) shape, the higher the probability of the respective observations corresponding to misclassification was.

has a normal equation

$$
\left(X^{\prime} X\right) b=X^{\prime} Y
$$

which is equal to

$$
\left[\begin{array}{ccc}
n & \sum X_{i 1} & \sum X_{i 2} \\
\sum X_{i 1} & \sum X_{i 1}^{2} & \sum X_{i 1} X_{i 2} \\
\sum X_{i 2} & \sum X_{i 2} X_{i 1} & \sum X^{2}{ }_{i 2}
\end{array}\right]\left[\begin{array}{c}
b_{0} \\
b_{1} \\
b_{2}
\end{array}\right]=\left[\begin{array}{c}
\sum Y_{i} \\
\sum X_{i 1} Y_{i} \\
\sum X_{i 2} Y_{i}
\end{array}\right],
$$

and the following normal equations,

$$
\begin{aligned}
\sum Y_{i} & =n b_{0}+b_{1} \sum X_{i 1}+b_{2} \sum X_{i 2} \\
\sum X_{i 1} Y_{i} & =b_{0} \sum X_{i 1}+b_{1} \sum X_{i 1}^{2}+b_{2} \sum X_{i 1} X_{i 2} \\
\sum X_{i 2} Y_{i} & =b_{0} \sum X_{i 2}+b_{1} \sum X_{i 1} X_{i 2}+b_{2} \sum X_{i 2}^{2}
\end{aligned}
$$

are obtained. In this case, the equations are

$$
\begin{aligned}
& X_{i 1}=i, \quad i=1,2,3,4, \\
& X_{i 2}=i^{2}, \quad i=1,2,3,4, \\
& Y_{1}=Z_{(1)}, \\
& Y_{2}=Z_{(3)}, \\
& Y_{3}=Z_{(4)}, \\
& Y_{4}=Z_{(2)} .
\end{aligned}
$$

Now, if

$$
\begin{aligned}
& S_{1}=Z_{(1)}+Z_{(2)}+Z_{(3)}+Z_{(4)}, \\
& S_{2}=Z_{(1)}+2 Z_{(3)}+3 Z_{(4)}+4 Z_{(2)}, \\
& S_{3}=Z_{(1)}+4 Z_{(3)}+9 Z_{(4)}+16 Z_{(2)},
\end{aligned}
$$




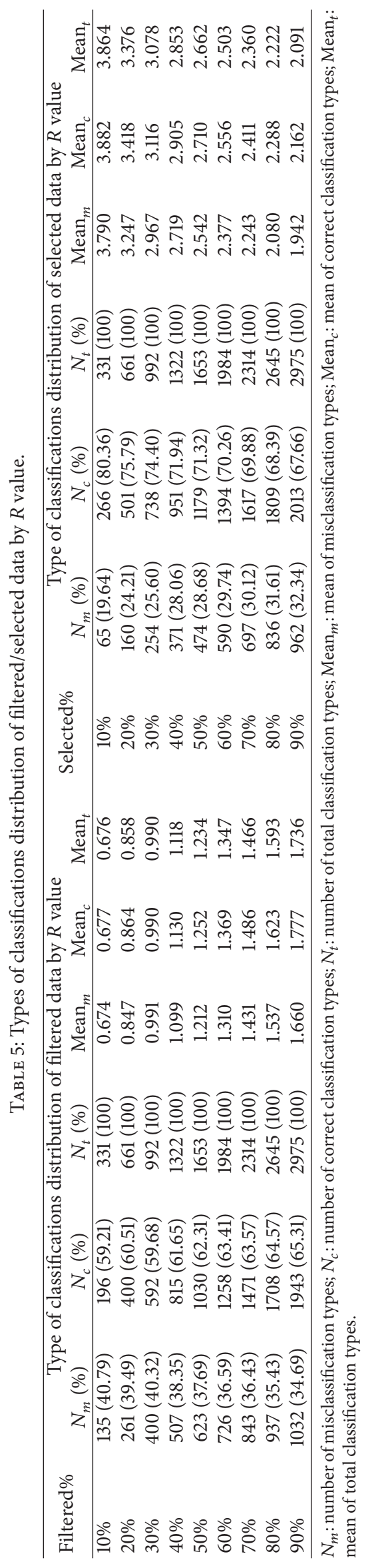




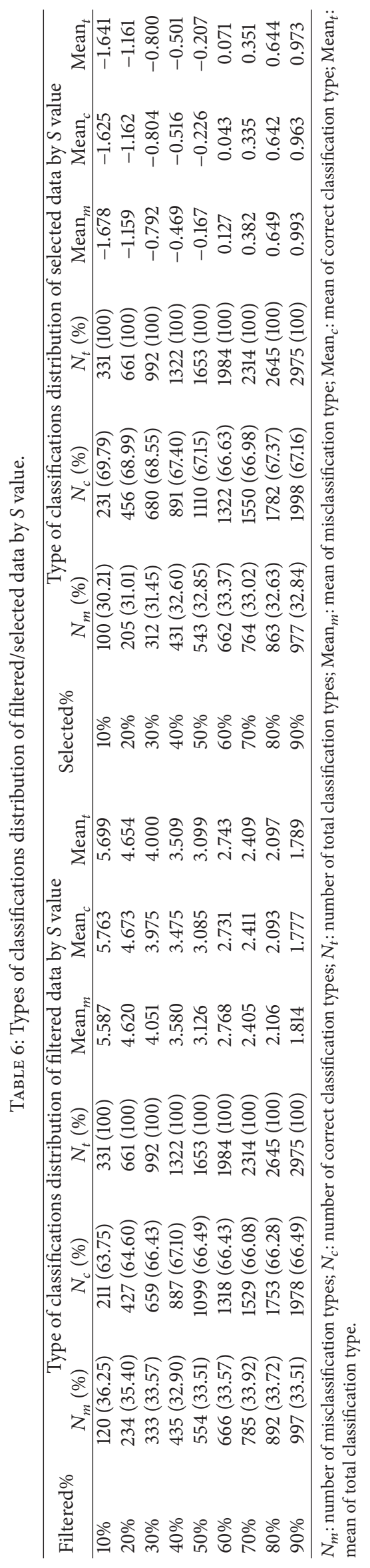




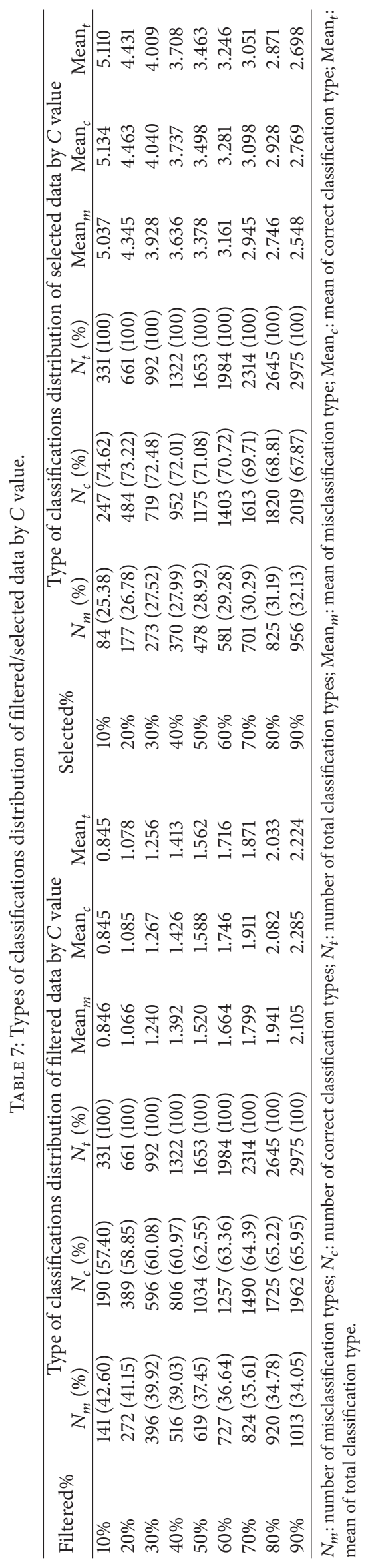




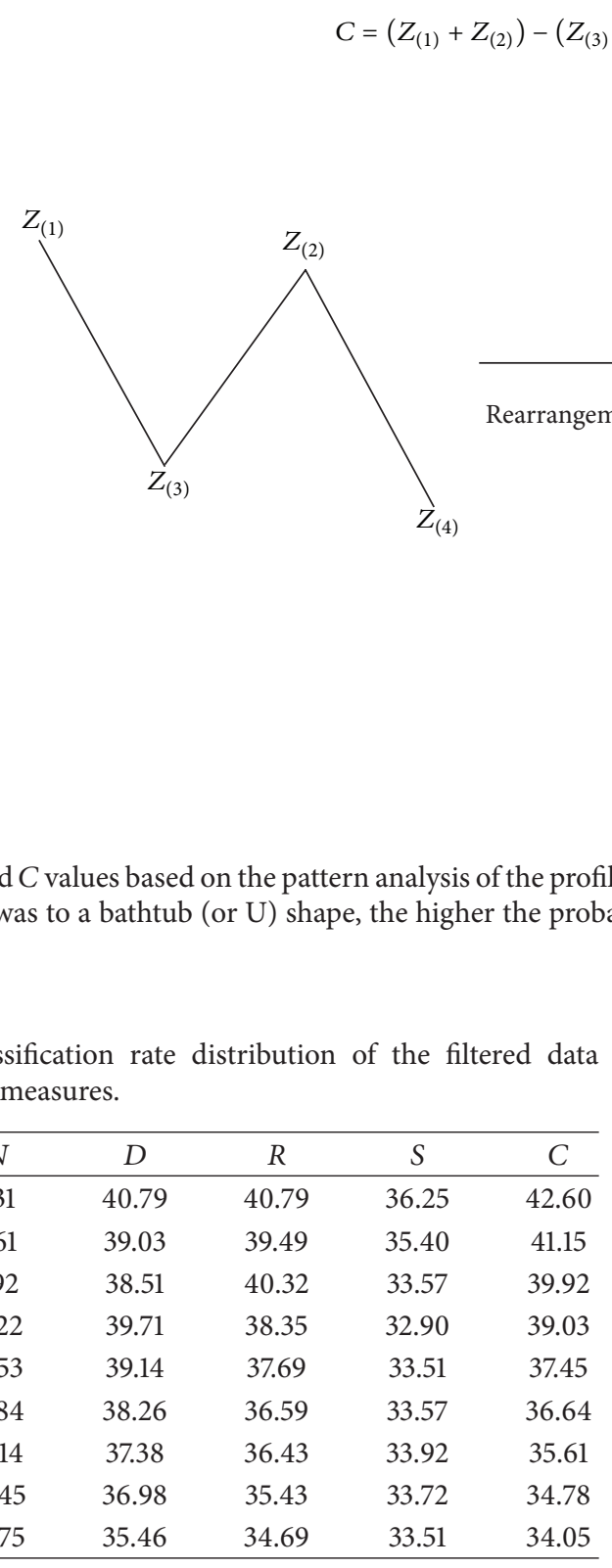

the normal equations should be equal to

$$
\begin{aligned}
& S_{1}=4 b_{0}+10 b_{1}+30 b_{2}, \\
& S_{2}=10 b_{0}+30 b_{1}+100 b_{2}, \\
& S_{3}=30 b_{0}+100 b_{1}+354 b_{2}
\end{aligned}
$$

and, ultimately, we obtain

$$
\begin{aligned}
\therefore b_{2} & =\frac{5}{4}\left(S_{1}-S_{2}+\frac{S_{3}}{5}\right) \\
& =\frac{1}{4}\left\{\left(Z_{(1)}+Z_{(2)}\right)-\left(Z_{(3)}+Z_{(4)}\right)\right\} .
\end{aligned}
$$

Certainly, the values of $b_{0}$ and $b_{1}$ may be obtained but omitted herein because they are meaningless. In (10), $Z_{(1)}$ and $Z_{(2)}$ are symmetric, and so are $Z_{(3)}$ and $Z_{(4)}$. Namely, when the curvature creates the largest profile with the 4 points, the curvature will not have any changes even if the largest and the second largest scores were switched. This also holds true for the smallest and the second smallest scores.

In the meantime, the $b_{2}$ value equals $1 / 4$ of the $C$ score among the 4 criteria obtained. Namely, the previously used $C$ score was equal to $Z_{(3)}$ and $Z_{(4)}$ was simply subtracted from the total of $Z_{(1)}$ and $Z_{(2)}$, which was the same as the secondary curvature created by the 4 scores.

\section{Discussion}

In TKM, a PI diagnostic system-one of the core technologies in the diagnosis and treatment of oriental medicineis used to determine the cause and nature of a disease, treatment methods, and treatment drugs for the patients [57]. However, the PI diagnosis holds limited objectivity and reproducibility due to the lack of standardized measurement indices. Objectification problems have always arisen with respect to personal deviations among TKM physicians. As the demand for the reestablishment and development of TKM has increased, studies on the establishment of a scientific basis for and the standardization of PI have been actively conducted $[7,12]$.

In this study, the clinical data of PI diagnosis for stroke were used to analyze and quantify the profile patterns of the misclassification types by applying the proposed scores to the comparative analysis. This was intended to boost the correct classification of objects by detecting those objects with a high probability of actual misclassification and deferring discrimination. Misclassification types were discerned by a discriminant analysis on the actual clinical data of PI diagnosis for stroke and quantified by a profile pattern analysis. The 
TABLE 9: Discriminant rate distribution of the selected data according to four measures.

\begin{tabular}{|c|c|c|c|c|c|}
\hline & \multicolumn{5}{|c|}{ Discriminant rate } \\
\hline & $N$ & $D$ & $R$ & $S$ & C \\
\hline $100 \%$ & 3306 & 66.82 & 66.82 & 66.82 & 66.82 \\
\hline $90 \%$ & 2975 & $68.24(+1.42)$ & $67.63(+0.81)$ & $66.92(+0.10)$ & $67.53(+0.71)$ \\
\hline $80 \%$ & 2645 & $68.62(+0.38)$ & $68.47(+0.84)$ & $67.15(+0.23)$ & $69.04(+1.51)$ \\
\hline $70 \%$ & 2314 & $69.53(+0.91)$ & $69.97(+1.50)$ & $66.98(-0.17)$ & $69.49(+0.45)$ \\
\hline $60 \%$ & 1984 & $71.98(+2.45)$ & $70.82(+0.85)$ & $66.94(-0.04)$ & $71.22(+1.73)$ \\
\hline $50 \%$ & 1653 & $73.32(+1.34)$ & $73.08(+2.26)$ & $69.03(+2.09)$ & $71.81(+0.59)$ \\
\hline $40 \%$ & 1322 & $75.34(+2.02)$ & $74.28(+1.20)$ & $68.68(-0.35)$ & $73.75(+1.94)$ \\
\hline $30 \%$ & 992 & $77.32(+1.98)$ & $76.81(+2.53)$ & $70.26(+1.58)$ & $75.81(+2.06)$ \\
\hline $20 \%$ & 661 & $83.36(+6.04)$ & $80.94(+4.13)$ & $73.83(+3.57)$ & $77.61(+1.80)$ \\
\hline $10 \%$ & 331 & $89.12(+5.76)$ & $87.01(+6.07)$ & $75.83(+2.00)$ & $82.78(+5.17)$ \\
\hline
\end{tabular}

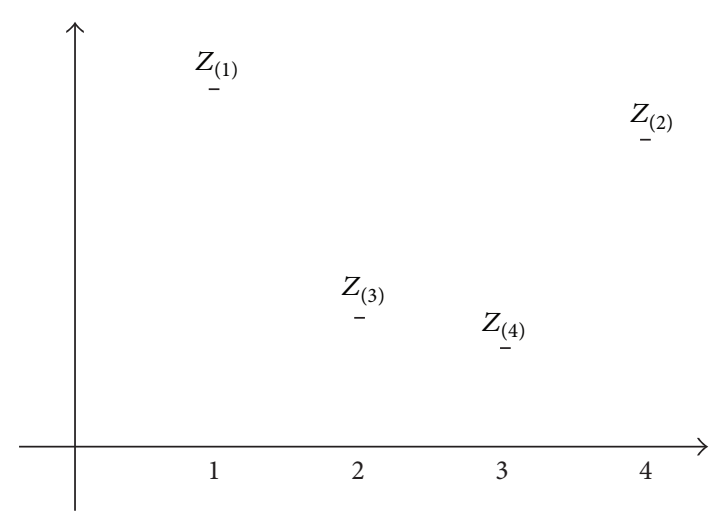

FIGURE 13: Curvature created by $Z$ scores $\left(Z_{(1)}, Z_{(2)}, Z_{(3)}\right.$, and $\left.Z_{(4)}\right)$. $Z_{(1)}, Z_{(2)}, Z_{(3)}$, and $Z_{(4)}$, as dependent variables observed in the $x$ values having equal intervals. $Z_{(1)}$ is a dependent variable when $x=$ $1, Z_{(2)}$ when $x=4, Z_{(3)}$ when $x=2$, and $Z_{(4)}$ when $x=3$.

proposed criteria of each standard were applied to the data already discriminated by the previous discriminant analysis in order to compare how well the misclassification had been estimated and how much the discrimination rate had improved when the estimated misclassification observations were removed in advanced. Particularly, the $C$ score delivered the same results as those from the discrimination of misclassification observations through a secondary curvature. Going forward, the following studies must be performed. First of all, 4 criteria to estimate misclassification were proposed in this study and applied to the actual clinical data, producing the possibility of better estimation of partial misclassification. Nonetheless, it was difficult to notably enhance discrimination rates and additional research appears to be necessary. In addition, 4 pattern groups with a different sample size were used in this study. Hence, the effects of different sample sizes need to be investigated.

\section{Conflict of Interests}

The authors declare that there is no conflict of interests regarding the publication of this paper.

\section{Acknowledgment}

This research was supported by a grant from the Korea Institute of Oriental Medicine (K13130, K16111).

\section{References}

[1] Social Statistics Office, 2012 Statistics on the Aged, Korean Statistical Information Service, Seoul, Republic of Korea, 2012.

[2] H. S. Jeong, Progress of Aging Population and Policy Responses in Japan, Research Department, The Bank of Korea, Seoul, South Korea, 2007 (Korean), http://public.bokeducation.or.kr/ecostudy/ publishList.do \&bbsId=22\&mode $=$ view\&contentId=2655\&cPage $=19$.

[3] Social Statistics Office, Statistics on the Cause of Death, Korean Statistical Information Service, Seoul, Republic of Korea, 2010.

[4] Division of Statistical Analysis in the Institute of Health Insurance Policy, The Status of Health Insurance Benefits of the Inpatients Frequent Diseases on Classified Subdivision of Diseases in Traditional Korean Medicine 2009, The National Health Insurance Corporation, Seoul, Republic of Korea, 2010.

[5] WHO Western Pacific Region, WHO International Standard Terminologies on Traditional Medicine in the Western Pacific Region, World Health Organization (WHO), Geneva, Switzerland, 2007.

[6] S. S. Shin, S. M. Choi, M. K. Shin, and K. S. Yang, A Study of Standardization of Diagnoses and Diagnostic Requirements in Traditional Korean Medicine III, Korea Institute of Oriental Medicine, Seoul, Republic of Korea, 1997.

[7] T.-Y. Park, J. A. Lee, M. H. Cha et al., “The fundamental study for the Standardization and Objectification of Pattern Identification in traditional Korean medicine for stroke (SOPIStroke): an overview of phase I," European Journal of Integrative Medicine, vol. 4, no. 2, pp. e125-e131, 2012.

[8] M. M. Ko, J. A. Lee, K. J. Yun, S. S. You, and M. S. Lee, "Perception of pattern identification in traditional medicine: a survey of Korean medical practitioners," Journal of Traditional Chinese Medicine, vol. 34, no. 3, pp. 369-372, 2014.

[9] J. K. Kim, I. C. Seol, I. Lee, K. Oh, B. C. Yu, and S. M. Choi, "Report on the Korean standard differentiation of the symptoms and signs for the stoke-1," Korean Journal of Oriental Physiology \& Pathology, vol. 20, pp. 229-234, 2006.

[10] H. Y. Go, Y. K. Kim, B. K. Kang et al., "Report on the Korean standard differentiation of the symptoms and signs for the 
stoke-2," Korean Journal of Oriental Physiology \& Pathology, vol. 20, pp. 1789-1791, 2006.

[11] J. A. Lee, J. S. Lee, M. M. Ko et al., "Report on the Korean standard pattern identifications for stroke-III," The Journal of Korean Oriental Internal Medicine, vol. 32, pp. 232-242, 2011.

[12] J. A. Lee, M. H. Cha, B.-K. Kang et al., "Fundamental study for the standardization and objectification of pattern identification in traditional Korean medicine for stroke (SOPI-Stroke): an overview of the second and third stages," European Journal of Integrative Medicine, vol. 7, no. 4, pp. 378-383, 2015.

[13] B.-K. Kang, T.-W. Moon, J. A. Lee, T.-Y. Park, M. M. Ko, and M. S. Lee, "The fundamental study for the standardisation and objectification of pattern identification in traditional Korean medicine for stroke (SOPI-Stroke): development and interobserver agreement of the Korean standard pattern identification for stroke (K-SPI-Stroke) tool," European Journal of Integrative Medicine, vol. 4, no. 2, pp. e133-e139, 2012.

[14] I. C. Kim, M. H. Cha, D. M. Kim et al., "A functional promoter polymorphism $-607 \mathrm{G}>\mathrm{C}$ of WNT10B is associated with abdominal fat in Korean female subjects," Journal of Nutritional Biochemistry, vol. 22, no. 3, pp. 252-258, 2011.

[15] M. M. Ko, T.-Y. Park, J. H. Lim, M. H. Cha, and M. S. Lee, "WNT10B polymorphism in Korean stroke patients with Yin deficiency pattern," Evidence-Based Complementary and Alternative Medicine, vol. 2012, Article ID 798131, 6 pages, 2012.

[16] J. H. Lim, M. M. Ko, J. S. Lee et al., "Genetic association of SNPs located at PON1 gene with dampness and phlegm pattern identification among Korea stroke patients," The Journal of Korean Oriental Internal Medicine, vol. 31, pp. 752-762, 2010.

[17] B. K. Kang, K. W. Kang, S. W. Park et al., "The discrimination model for the pattern identification diagnosis of the stroke," Korean Journal of Oriental Internal Medicine, vol. 13, no. 2, pp. 59-63, 2007.

[18] B. K. Kang, J. S. Lee, S. Y. Kim et al., "The discrimination model IV for syndrome differentiation diagnosis in stroke patients," Journal of the Korean Data Analysis Society, vol. 11, no. 6, pp. 2995-3007, 2009.

[19] B. K. Kang, M. M. Ko, J. A. Lee, T. Y. Park, and Y. G. Park, "Discriminant modeling for pattern identification using the Korean standard PI for stroke-III," Korean Journal of Oriental Physiology \& Pathology, vol. 25, no. 6, pp. 1-6, 2011.

[20] J. A. Lee, T.-Y. Park, J. S. Lee et al., "Developing indicators of pattern identification in patients with stroke using traditional Korean medicine," BMC Research Notes, vol. 5, article 136, 2012.

[21] J. Neter, W. Wasserman, and M. H. Kutner, Applied Linear Statistical Models, CRC Press, Philadelphia, Pa, USA, 3rd edition, 1990. 


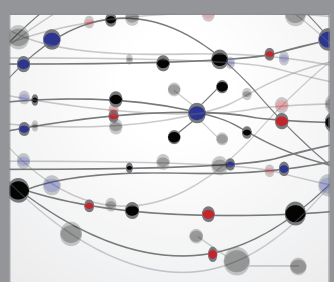

The Scientific World Journal
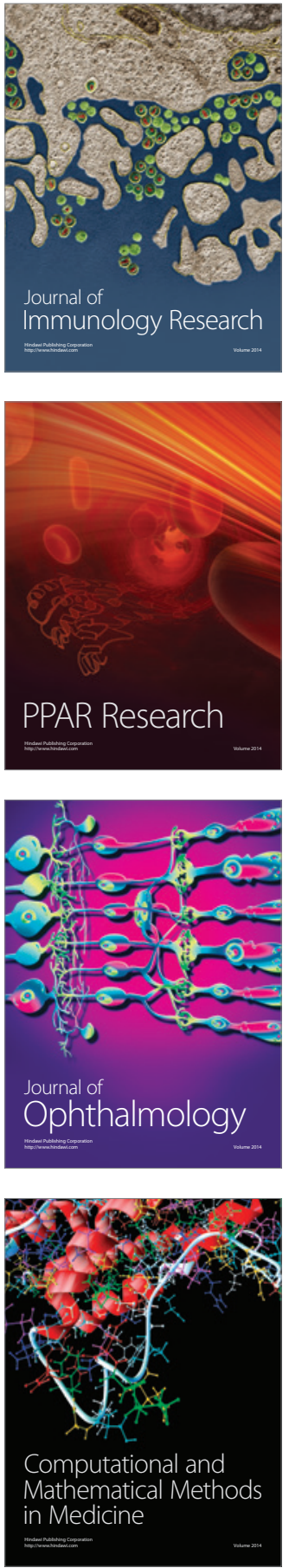

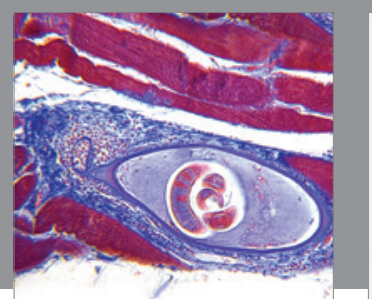

Gastroenterology Research and Practice

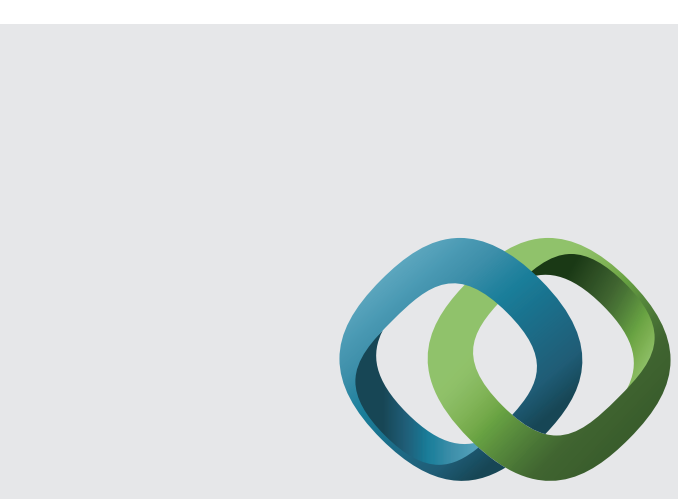

\section{Hindawi}

Submit your manuscripts at

http://www.hindawi.com
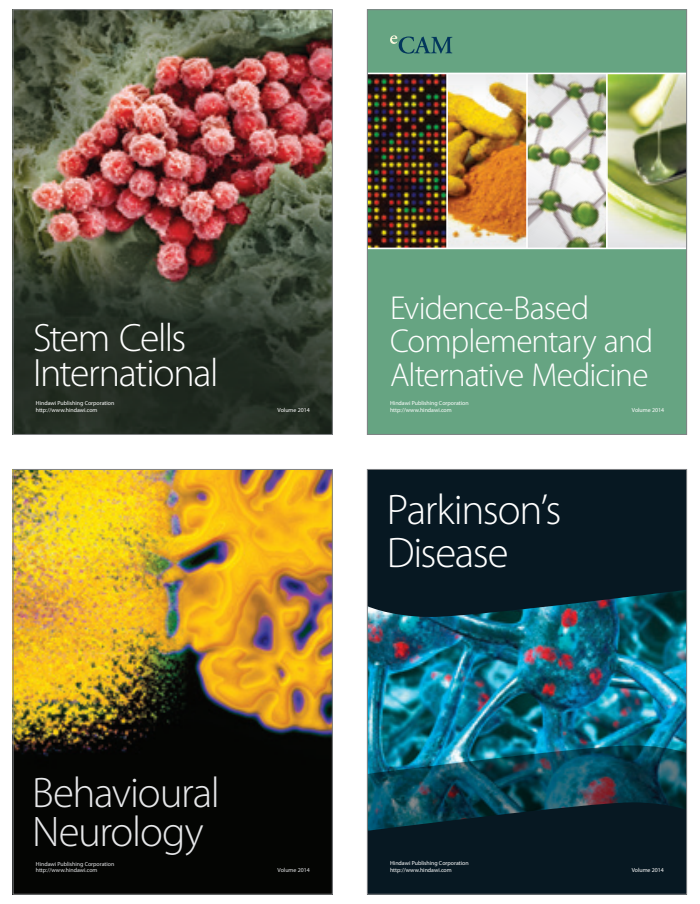
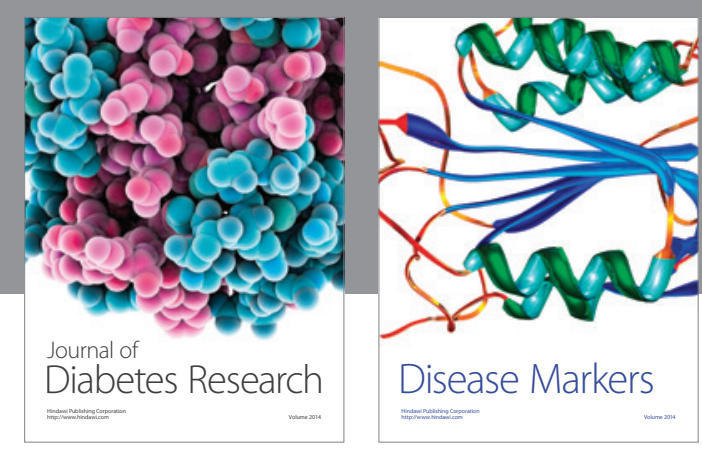

Disease Markers
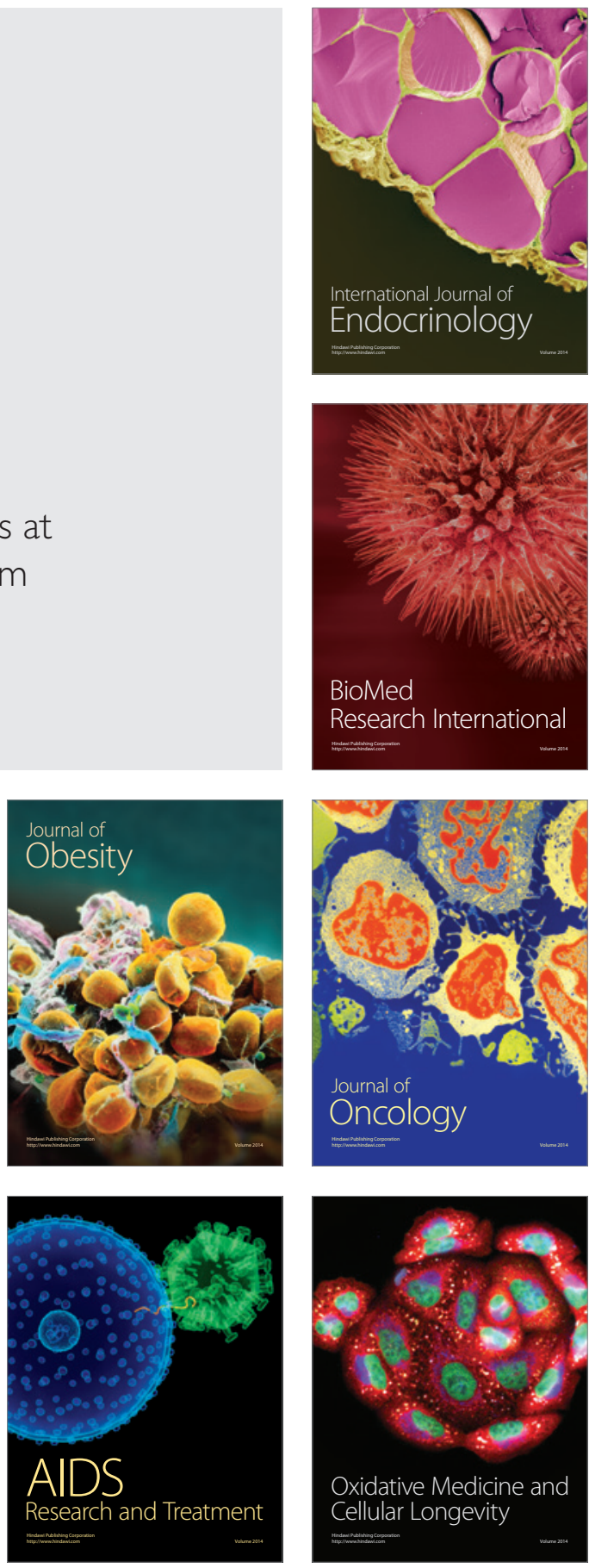